Supplementary Materials for

\title{
Electrically Biased Silicon Metasurfaces with Magnetic Mie Resonance for Tunable Harmonic Generation of Light
}

Kyu-Tae Lee, ${ }^{1+}$ Mohammad Taghinejad, ${ }^{1+}$ Jiahao Yan, ${ }^{1+}$ Andrew S. Kim, ${ }^{1}$ Lakshmi Raju, ${ }^{1}$ Devin K. Brown ${ }^{1}$ and Wenshan Cai ${ }^{* 1,2}$

${ }^{1}$ School of Electrical and Computer Engineering, Georgia Institute of Technology, Atlanta, Georgia 30332, USA

${ }^{2}$ School of Materials Science and Engineering, Georgia Institute of Technology, Atlanta, Georgia 30332, USA

*E-mail: wcai@gatech.edu

† K-T.L., M. T., J. Y. contributed equally to this work

Keywords: Metamaterials, Nonlinear optics, Mie resonance, Silicon photonics, Optoelectronics 


\section{Methods}

\section{Detailed fabrication process}

(a)

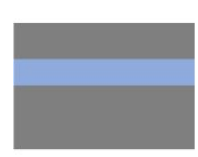

(e)

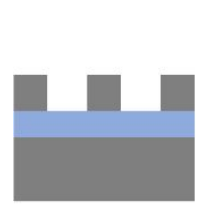

(b)

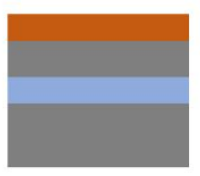

(f)

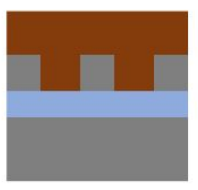

(c)

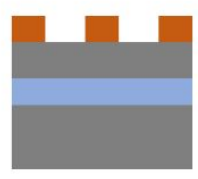

(g)

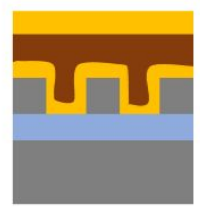

(d)

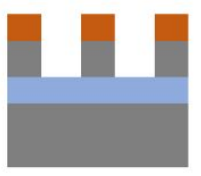

(h)
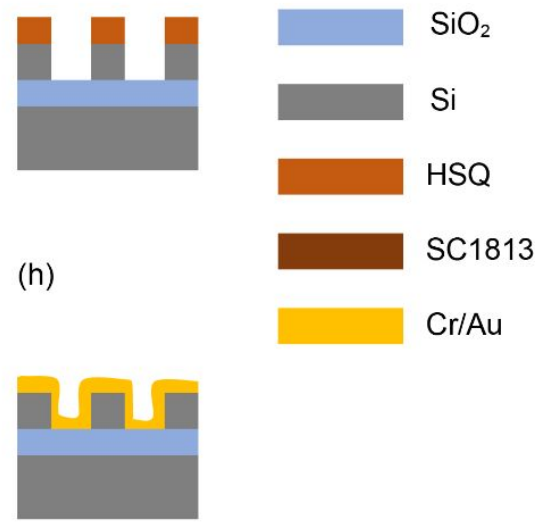

(i)

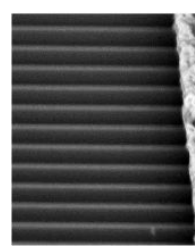

(j)
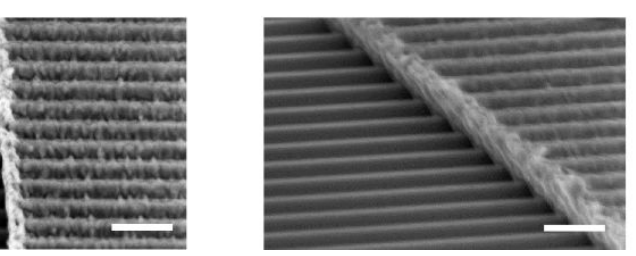

Figure S1 Fabrication flowchart of the Si metasurfaces with electric contacts. The scale bars represent $500 \mathrm{~nm}$.

The fabrication flowchart is illustrated in Figure S1. (a) The silicon metasurfaces are fabricated on the silicon on insulator (SOI) wafer (Solitec). The SOI wafer is composed of $190 \mathrm{~nm}$ of (100) silicon on top of $150 \mathrm{~nm}$ of $\mathrm{SiO}_{2}$ layer. (b) A $115 \mathrm{~nm}$ of negative e-beam resist (XR-1541, $6 \%$ HSQ) is formed on top of the SOI wafer by spin coating the resist at 3200 RPM for 60 seconds with ramp time of 2 seconds followed by a pre-baking process at $80{ }^{\circ} \mathrm{C}$ for $4 \mathrm{~min}$. The resist is selected to form a hard mask to etch the top silicon layer, as it turns into silicon dioxide after the full EBL process is performed. (c) Next, electron beam lithography (Elionix ELS-G100 EBL system) is carried out to define the geometry of the silicon metasurface. In this step, the alignment 
marks are also defined, which is necessary for the photolithography process. After the EBL exposure, the resist is developed in $25 \%$ Tetramethylammonium hydroxide (TMAH) solution for $30 \mathrm{~s}$ under $80{ }^{\circ} \mathrm{C}$ and rinsed gently by deionized water to reduce residue. Then the remaining ebeam resist structure turns into silicon dioxide which can serve as a hard mask during the etching process (d) The exposed area of the single crystalline silicon layer is etched by inductively coupled plasma - reactive ion etching (ICP-RIE) technique using the chlorine $\left(\mathrm{Cl}_{2}\right)$ gas. The etch rate of the silicon is measured as $\sim 12 \AA / \mathrm{s}$, where the etch selectivity between the Si and HSQ hard mask is 4 to 1 . By controlling the pressure, coil and platen power, we could achieve a vertical sidewall of the silicon strips. (e) Hard mask (HSQ) is removed by dipping the sample into the buffered oxide etchant (6:1) for 1 2 seconds and quickly rinsed by DI water. This step is conducted carefully and precisely to prevent etching the buried $\mathrm{SiO}_{2}$ layer in the $\mathrm{SOI}$ wafer during the process. (f) Next, standard photolithography technique is conducted to define the electrodes. Positive photoresist (SC1813) of $1.7 \mu \mathrm{m}$ in thickness is spin-coated at $3000 \mathrm{RPM}$ with ramp time of 2 seconds for 60 seconds and it is prebaked at $115^{\circ} \mathrm{C}$ for 50 seconds. Then the photoresist layer was exposed (Karl Suss mask aligner) and developed by Microposit MF-319 (MicroChem) developer. (g) Afterwards, $100 \AA$ and $2500 \AA$ thick $\mathrm{Cr}$ and Au layer is deposited by using e-beam evaporator (CHA Industries, Inc.), respectively, where the deposition rate for the both processes is fixed at $0.5 \AA / s$. (h) Subsequently, a lift-off process using the Acetone solution is carried out to form a metal electrode for the silicon metasurfaces. (i) and (j) show the top and side views of the fabricated device, respectively. It shows that the silicon gratings are well covered by the top gold electrode layer. 


\section{$\underline{\text { Linear optical characterization }}$}

The linear characterization for the fabricated silicon device is performed at a home-made experimental setup (not shown). A fiber coupled tungsten halogen lamp (B\&W Tek BPS 120) is used as a broadband light source to characterize the linear response of the silicon device. The polarization of the source is controlled by employing a set of a linear polarizer and half waveplate to generate TE- and TM-polarized light. The analysis is performed in reflection mode using beam splitters and optics. An objective lens (Mitutoyo, 20X plan Apo NIR infinity-corrected) is used to focus the incident light onto the sample and collect the reflected light from the sample. The collected light is then delivered to the spectroscopy system which is consist of a monochromator (Princeton Instrument Acton SP 2300i) with the visible-near IR detector (PIXIS 400B camera). The reflectance of the silicon metasurfaces is achieved by normalizing the reflection from that of the plane gold electrode as shown in Figure S2.

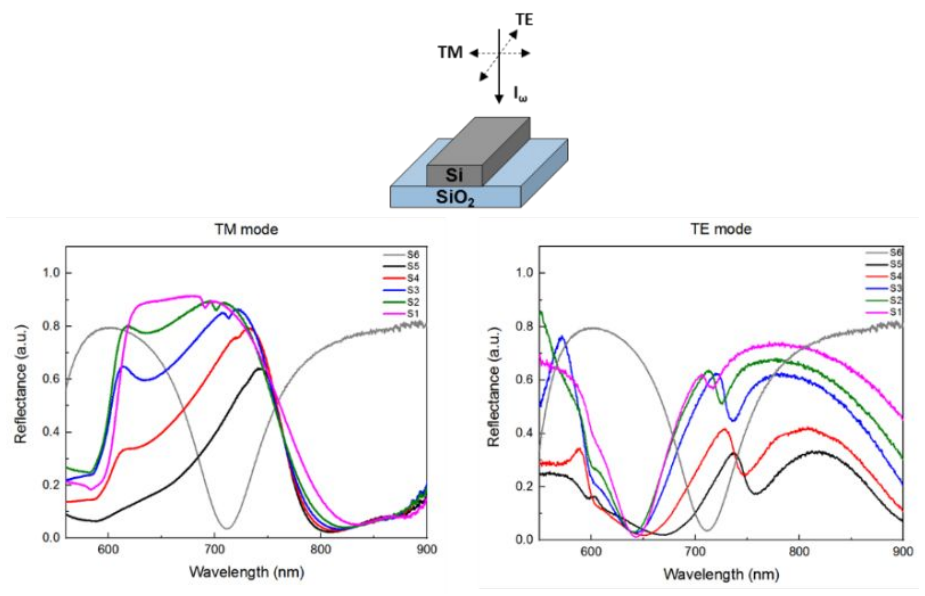

Figure S2 Reflectance from the six silicon devices at the both TE and TM polarization incidence. 


\section{$\underline{\text { Nonlinear optical characterization }}$}

A schematic of the home-mad experimental setup for nonlinear optical characterization is shown in Figure S3a. The fundamental wave is generated from a tunable Ti: Sapphire ultrafast oscillator (Spectra-Physics, Mai Tai HP, 690 1040 nm) with 100 femtosecond pulse duration and $80 \mathrm{MHz}$ repetition rate. The power and the polarization states of the incident light are controlled by the set of halfwave plates and the Glan polarizer. The fundamental light passes through the beam splitter and focused on the sample by the objective lens $(20 \mathrm{x}, \mathrm{NA}=0.5)$. Both the fundamental and harmonic generated signals are collected by the objective and reflected by a beam splitter. The bandpass filter eliminates the fundamental wave so that only generated signal could be characterized by the detection system which is consists of a monochromator (Princeton Instruments, IsoPlane) with CCD camera (Princeton Instruments, Pixis 400B). The generated signal is collected with/without biasing the sample which the effect of static field induced SHG signal is analyzed by further data processing.

The focused beam has a spot size of $20 \sim 40 \mu \mathrm{m}$ depending on the intensity of the incident beam so that it could excite the silicon gratings while minimizing the nonlinear effect from the gold electrode (see Figure S3a). Though the beam size is smaller than the width of the exposed silicon grating area, the comparison of acquired spectra of the SHG signals between the gold pad and the device is performed to further elucidate the effect of the SHG signal from the gold electrode on the total SHG signal from the device S3 (see Figure S3b). For the measurement, TM-polarized laser pulses of varying fundamental wavelengths from 720 to $900 \mathrm{~nm}$ are used to excite the pad and device at a constant intensity. As shown in Figure S3b, the SHG signal acquired from the gold pad was negligible compared to the SHG signal observed from the silicon grating, from which we can safely assume that the signal from the gold pad can be ignored. 
a

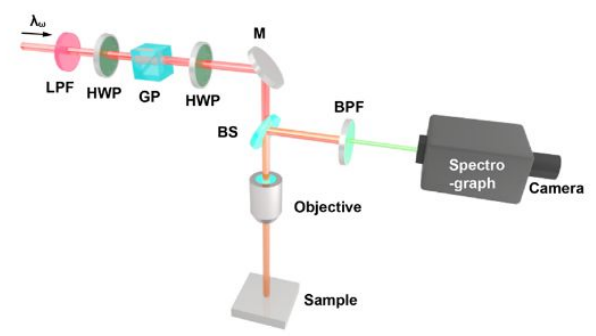

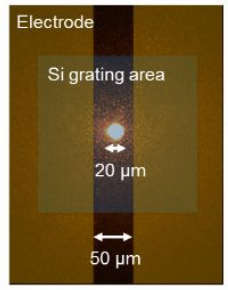

b

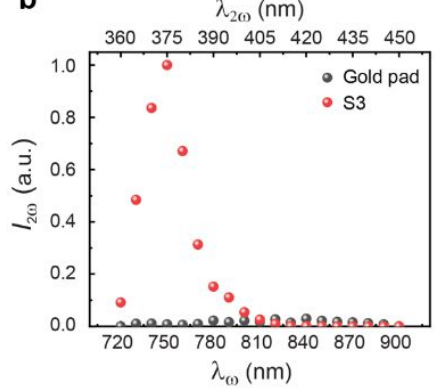

Figure S3 (a) Experimental setup for characterizing SHG / EFISH signal from the device. Abbreviation for optical components: LPF: long-pass filter; HWP: half waveplate; GP: GlanPolarizer; M: mirror; BS: Beam splitter; BPF: band-pass filter. (b) Comparison of the second harmonic signal from the gold pad and device S3 at a series of excitation wavelengths of a constant intensity. 


\section{Polarized excitation analysis}

Figure S4a illustrates the polarization survey of the S3 device to investigate the behavior of the magnetic Mie resonance due to the polarization of the illuminating light. The input polarization of the light is controlled by the halfwave plate as described above in the "Linear optical characterization" section. At the expected spectral location of the magnetic Mie resonance, at $750 \mathrm{~nm}$, the TE polarized light exhibits lower reflectance than the TM polarized light. However, the cross-sectional view of the calculated field profile inside the silicon strips of the S3 device shows a stronger field is confined in the silicon stripes under TM polarization excitation as shown in Figure S4b. This is because the silicon stripes experience a strong coupling with TM polarized light and form a circulating electric field in the grating. This indicates that the magnetic Mie resonance is the source of the strong field enhancement in the silicon stripes which is dependent on the polarization of the incident light.

a

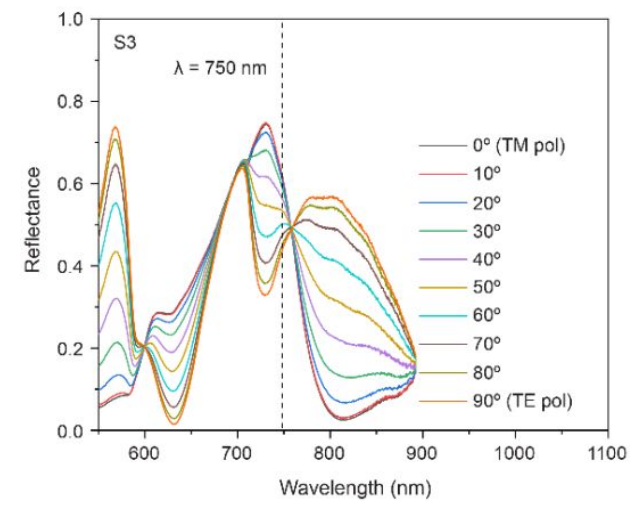

b

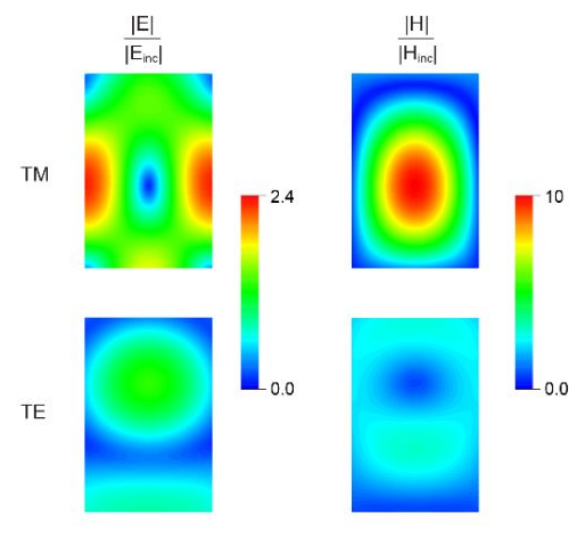

Figure S4 (a) Linear optical response of the S3 device obtained by varying the polarization of the incident light. (b) Calculated Cross-sectional views of the normalized electric and magnetic field distributions for the TE / TM polarization excitation at the wavelength $785 \mathrm{~nm}$. Strong field confinement is shown only under the TM polarization, which is induced by the magnetic Mie resonance. 


\section{Estimation of static SHG enhancement induced by the surface of the Si gratings $\left(\chi^{(2)}\right.$ tensor $)$}

Calculation of the static SHG is executed by incorporating the surface $\chi^{(2)}$ tensor of the (100) crystalline silicon ${ }^{1,2}$ and field enhancement originating from the magnetic Mie resonance. The bulk contribution to the SHG signal due to the high order multipoles, i.e., magnetic dipoles or electric quadrupoles, is neglected in this calculation due to its small contribution to the overall SHG intensity from the silicon structure. ${ }^{3}$ The nonlinear polarization at the surface $\left(P^{N L \text {, surf }}\right)$ for the (100) silicon is described as below:

$$
\left(\begin{array}{l}
P_{x}^{N L, \text { surf }} \\
P_{y}^{N L, \text { surf }} \\
P_{z}^{N L, \text { surf }}
\end{array}\right)=\left(\begin{array}{cccccc}
0 & 0 & 0 & 0 & d_{15} & 0 \\
0 & 0 & 0 & d_{15} & 0 & 0 \\
d_{31} & d_{31} & d_{33} & 0 & 0 & 0
\end{array}\right)\left(\begin{array}{c}
E_{x}^{2} \\
E_{y}^{2} \\
E_{z}^{2} \\
2 E_{y} E_{z} \\
2 E_{x} E_{z} \\
2 E_{x} E_{y}
\end{array}\right)
$$

As the devices are fabricated simultaneously on the same SOI wafer, the azimuthal dependency of the SHG output from the (100) silicon surface is ignored to simplify the calculation. From the cross-sectional view of the electric field vector profile shown at the main manuscript (Figure 3b), the silicon strips under the TM-polarized fundamental wavelength of $785 \mathrm{~nm}$ can possess electric field in both $\mathrm{x}$ and $\mathrm{z}$ directions, while the planar Si pad device possess electric field in only the $\mathrm{x}$ direction. Moreover, the electric field inside the strips is strongly confined so that it could further enhance the SHG signal from the grating surfaces.

Therefore, the contribution of the surface $\chi^{(2)}$ tensor can be analyzed based on the two cases: second harmonic signal generates 1) only from the $\mathrm{x}$-component and 2) from both $\mathrm{x}$ - and z-components of the electric field. By combining the nonlinear polarization tensor of the silicon 
surface, $P^{N L \text {, surf }}$, with the two cases, the total nonlinear polarization which contributes to the SHG signals of each cases are derived as:

$$
\begin{aligned}
& P_{\text {tot, } \mathrm{E}_{\mathrm{x}}}^{N L \text {, }}=P_{z}^{N L, \text { surf }}=d_{31} E_{x}^{2} \\
& P_{\mathrm{tot}, \mathrm{E}_{\mathrm{x}}, \mathrm{E}_{\mathrm{z}}}^{N L, \text { surf }}=P_{x}^{N L, \text { surf }}+P_{z}^{N L, \text { surf }}=2 d_{15} E_{x} E_{z}+d_{31} E_{x}^{2}+d_{33} E_{z}^{2} .
\end{aligned}
$$

The first case corresponds to the plane silicon sample, S6, where the second case describes the SHG behavior at the silicon metasurfaces. The value for $d_{15}, d_{31}$, and $d_{33}$ can be determined by a frequency-domain interferometric second harmonic generation technique. ${ }^{4}$ In this calculation, the $\mathrm{d}_{\mathrm{ij}}$ values are assumed to be positive and real so that $\mathrm{d}_{15} \sim 10^{-19}, \mathrm{~d}_{31} \sim 10^{-21}$, and $\mathrm{d}_{33} \sim 10^{-18}$ $\mathrm{m}^{2} / \mathrm{V}$ are estimated at the fundamental wavelength of $745 \mathrm{~nm}$. By assigning the $\mathrm{d}_{\mathrm{ij}}$ values to Eq. (2), we could find the significant role of $\mathrm{E}_{\mathrm{z}}$ components in the total nonlinear polarization as the magnitude of $d_{31}$ is negligible compare to the other two terms.

Next, we calculated the total field enhancement in the silicon metasurfaces, by combining two factors from the field enhancement in a single silicon rod due to the magnetic Mie resonance and the filling ratio of the silicon strips at the silicon metasurfaces. The filling ratio (FR) is defined by dividing the width of the grating $(130 \mathrm{~nm})$ to its periodicity $(\mathrm{S} 1: 280 \mathrm{~nm}, \mathrm{~S} 2: 330 \mathrm{~nm}$, S3: $380 \mathrm{~nm}, \mathrm{~S} 4: 430 \mathrm{~nm}, \mathrm{~S} 5: 480 \mathrm{~nm}$ ). Then the field enhancement and the nonlinear polarization is incorporated to estimate the SHG enhancement from the given silicon metasurfaces. Since the $E_{z}$ term at the surface of the silicon metasurfaces governs the total nonlinear polarization, only the electric field in z-direction at the surface of the grating is used for the SHG enhancement calculation. Figure S5 illustrates the cross-sectional view of the electric field profile of the proposed five metasurface device under TM polarized incidence at the fundamental wavelength of $785 \mathrm{~nm}$ and Table 1 illustrates the estimated SHG enhancement results from the contribution of $E_{z}$ field. High SHG enhancement is shown at the device S3 and $\mathrm{S} 4$ regardless of its low filling factors compare to S1 and S2. To explore the nonlinear response 
clearly at the spectral vicinity of the magnetic Mie resonance, the sample S3 is chosen whose magnetic Mie mode is spectrally distant from the reflection peak.

Table 1. SHG enhancement estimation based on the $E_{z}$ enhancement at the surface

\begin{tabular}{|c|c|c|c|c|}
\hline & P [nm] & FR & $\boldsymbol{E}_{\mathbf{z}}^{\mathbf{z}}$ enhancement & SHG enhancement (d $\left.\mathbf{d}_{\mathbf{3}}\right)$ \\
\hline S1 & 280 & 0.46 & 2.79 & 1.67 \\
\hline S2 & 330 & 0.39 & 4.21 & 2.75 \\
\hline S3 & 380 & 0.34 & 5.67 & 3.77 \\
\hline S4 & 430 & 0.30 & 6.44 & 3.79 \\
\hline S5 & 480 & 0.27 & 5.72 & 2.40 \\
\hline
\end{tabular}
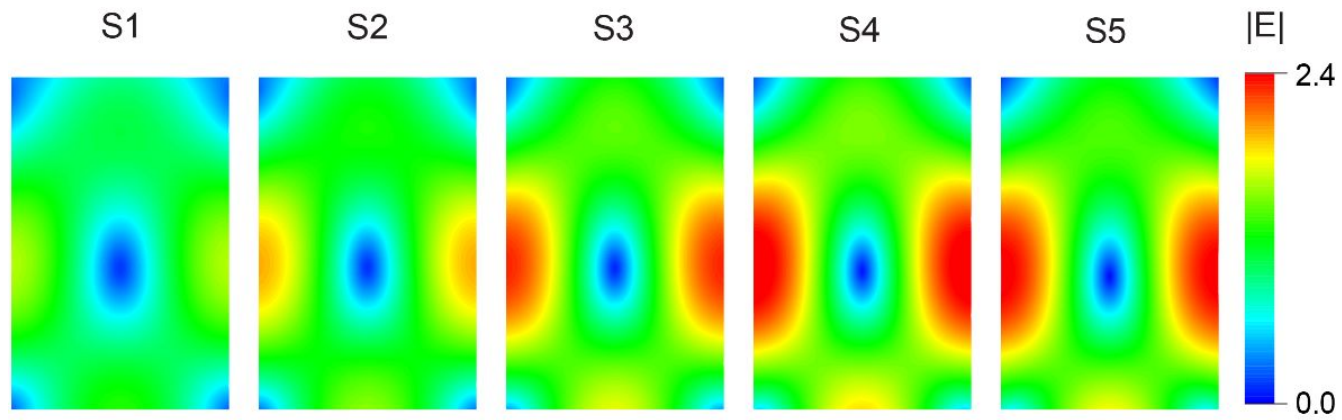

Figure S5 Electric field profile from the five devices at the TM polarized wavelength of $785 \mathrm{~nm}$ 


\section{Numerical calculation of the EFISH generation induced by magnetic Mie resonance with external static field $\left(\chi^{(3)}\right.$ tensor $)$}

EFISH generation from the device S3 is calculated numerically by combining the field profile at the fundamental wavelength $\lambda_{\omega}$, the static field induced by the external voltage, and the third-order susceptibility tensor $\left(\chi^{(3)}\right)$. As shown in Figure S4b, the field distribution in the silicon nano-stripes exhibits a strong dependence on the polarization of the incident light. When the silicon nanograting is excited by the TM polarized light, the structure could couple with the incoming wave which induces a circulating electric field inside the grating. This magnetic Mie mode indicates that the $\mathrm{x}$ - and $\mathrm{z}$ - components of the electric field dominate in the grating. On the other hand, the field profile shows that the field coupling between the silicon gratings and the TE polarized incident light is negligible compared to the TM polarized case.

Crystalline silicon belongs to the $\mathrm{m} 3 \mathrm{~m}$ symmetry group, where the third-order susceptibility tensor $\left(\chi^{(3)}\right)$ possesses 21 nonzero elements. Among the nonzero elements, only 4 are independent as shown below 5 :

$$
\begin{aligned}
& \mathrm{xxxx}=\mathrm{yyyy}=\mathrm{zzzz}, \quad \mathrm{yyzz}=\mathrm{zzyy}=\mathrm{zzxx}=\mathrm{xxzz}=\mathrm{xxyy}=\mathrm{yyxx}, \\
& \mathrm{yzyz}=\mathrm{zyzy}=\mathrm{zxzx}=\mathrm{xzxz}=\mathrm{xyxy}=\mathrm{yxyx} \\
& \mathrm{yzzy}=\mathrm{zyyz}=\mathrm{zxxz}=\mathrm{xzzx}=\mathrm{xzzx}=\mathrm{xyyx}=\mathrm{yxxy}
\end{aligned}
$$

As the static electric field induced by the external voltage forms the electric field along the silicon gratings $\left(E_{y}^{0}\right)$ and the dominant electric field direction induced by the magnetic Mie resonance are in the $\mathrm{x}$ - and z-direction, only two effective terms could contribute to the EFISH generation: $\chi_{y x x y}^{(3)}$ $E_{x}^{\omega} E_{x}^{\omega} E_{y}^{0}$ and $\chi_{y z z y}^{(3)} E_{z}^{\omega} E_{z}^{\omega} E_{y}^{0}$ 
Using the two effective terms as a source for the EFISH generation in the silicon gratings, a nonlinear response at device S3 is calculated as shown in Figure S6. Figure S6a shows the EFISH generated signal for both TE / TM polarized incident fundament light. The result clearly shows that the TM polarized light could produce the second-order signal effectively, thanks to the field localization and enhancement facilitated by the strong magnetic Mie resonance.

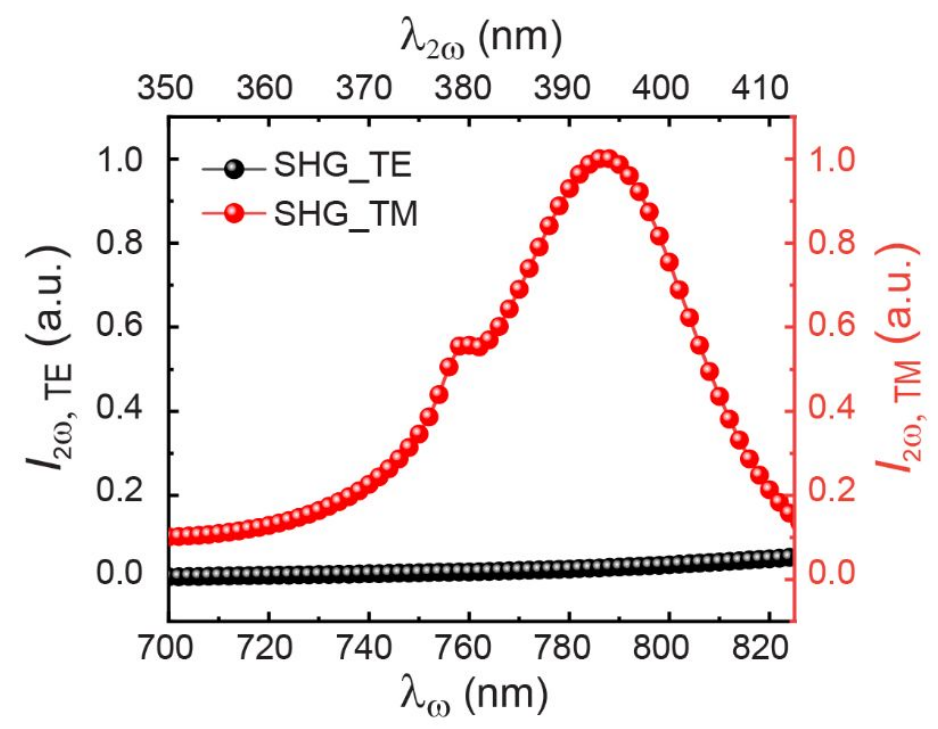

Figure S6 Numerical results for the EFISH generation from the S3 device for TE / TM polarized fundamental light. 


\section{Output polarization analysis of the EFISH signals}

Figure S7 shows the calculated and experimental results of EFISH generation from the $\mathrm{S} 3$ device. As described in the manuscript two effective terms, $\chi_{y x x y}^{(3)} E_{y}^{0}$ and $\chi_{y z z y}^{(3)} E_{y}^{0}$, are derived by incorporating the third-order nonlinear susceptibility tensor of single crystalline silicon and all involved electric field components in the silicon structure. The EFISH generation results exhibit in Figure S7a clearly shows that the SHG signal is generated along the silicon strips. Therefore, the EFISH generated signal is expected to possess polarization in the $\mathrm{y}$-direction. Figure S7b shows the experimental results of the output polarization of second-order nonlinear signal in the device S3. The intensity of the y-polarization from the voltage induced SHG (red dot) is shown to have higher value compared to the surface SHG (black dot) which indicates that the polarization of the EFISH generated signal contains a y-direction polarization.
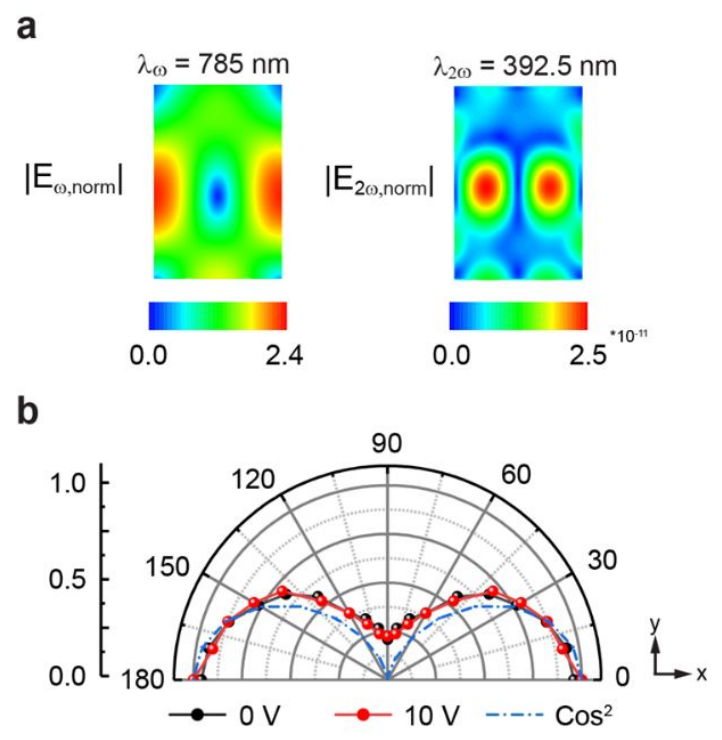

Figure S7 (a) Calculated cross-sectional view of the field distribution for both fundamental wavelength of $785 \mathrm{~nm}$ and second harmonic generation wavelength of $392.5 \mathrm{~nm}$ at sample S3. (b) Output polarization of SHG signal with / without applying the voltage at the S3 device. 


\section{REFERENCES}

1. Cazzanelli, M.; Schilling, J. Second order optical nonlinearity in silicon by symmetry breaking. J. Appl. Phys. Rev. 2016, 3 (1), 011104.

2. Sipe, J. E.; Moss, D. J.; van Driel, H. M. Phenomenological theory of optical second- and thirdharmonic generation from cubic centrosymmetric crystals. Phys. Rev. B Condens. Matter. 1987, 35 (3), 1129-1141.

3. Timbrell, D.; You, J. W.; Kivshar, Y. S.; Panoiu, N. C. A comparative analysis of surface and bulk contributions to second-harmonic generation in centrosymmetric nanoparticles. Sci. Rep. 2018, 8, 3586.

4. An, Y. Q.; Carriles, R.; Downer, M. C. Absolute phase and amplitude of second-order nonlinear optical susceptibility components at Si(001) interfaces. Phys. Rev. B 2007, 75 (24), 241307.

5. Boyd, R. W. Nonlinear Optics, 3rd Edition. Nonlinear Optics, 3rd Edition 